\title{
Discovery of anti-influenza nucleoside triphosphates targeting the catalytic site of $\mathrm{A} / \mathrm{PR} / 8 / 34 / \mathrm{H} 1 \mathrm{~N} 1$ polymerase
}

\author{
Nataraj Sekhar Pagadala $\mathbb{I}^{1,2} \cdot$ Rakesh $^{\text {Bhat }}{ }^{3} \cdot$ Jagadeesh Kumar $D^{4} \cdot$ Abdolamir Landi $^{1,2}$
}

Received: 8 December 2019 / Accepted: 12 May 2020 / Published online: 24 May 2020

(c) Springer Science+Business Media, LLC, part of Springer Nature 2020

\begin{abstract}
In an effort to develop potent anti-influenza drugs that inhibit the activity of influenza virus RNA-dependent RNA polymerase (IAV RdRp), a database of nucleoside triphosphates with $\sim 800$ molecules were docked with the homology model of IAV RdRp from A/PR/8/34/H1N1 strain. Out of top 12 molecules that bind with higher affinities to the catalytic site of IAV RdRp above and below the PB1 priming loop, only seven molecules decreased the transcriptional activity of the viral RNA polymerase with an $\mathrm{IC}_{50}$ in the range of $0.09-3.58 \mu \mathrm{M}$. Molecular docking combining with experimental study indicated that the molecules with linear chain are more effective in inhibiting IAV RdRp replication than the molecules with V-shaped and are cyclic in nature. A correlation between $\Delta G$ and $\operatorname{LogIC}_{50}$ for these seven compounds resulted an $R^{2}$ value of 0.73 . Overall, these newly developed seven nucleoside triphosphates lay a strong foundation for the future development of a new therapeutics that can satisfy the Lipinski's rule of five exhibiting high specificity to the catalytic site of influenza-A viruses.
\end{abstract}

Keywords RNA-dependent RNA polymerase $\cdot$ Catalytic site $\cdot$ Docking $\cdot$ Nucleoside triphosphates

\section{Introduction}

Influenza is a highly contagious airborne life-threatening viral infection causing recurrent outbreaks of humans responsible for respiratory diseases and death. It was estimated during 2017-2018 that there were 959,000 hospitalizations and 79,400 deaths (CDC 2018) alone in the United States related to influenza illness. Moreover, WHO estimates that 3-5 million people suffer from the disease every year with death rate ranging between 290,000 and 650,000 due to influenza epidemics (Influenza (Seasonal) 2018) worldwide (Sherman et al. 2019). Of note, previous epidemiological studies on pandemic $\mathrm{A} / \mathrm{H} 1 \mathrm{~N} 1$ during the year

Nataraj Sekhar Pagadala

nattu251@gmail.com

1 Department of Medical Microbiology and Immunology, University of Alberta, Edmonton, AB T6G 2E1, Canada

2 Li Ka Shing Institute of Virology, University of Alberta, Edmonton, AB, Canada

3 Precision Bio Laboratories, Edmonton, AB, Canada

4 Department of Biotechnology, Sir M. Visvesvaraya Institute of Technology, Bangalore, India
2009 clearly showed that the severity of influenza was not so high in individuals effected with HIV compared with HIV-negative patients (Martinez et al. 2011; Perez et al. 2010). This clearly shows that influenza remains a major target for vaccine and antiviral treatment and prophylaxis. One of the causes of the severity of the disease is due to influenza replication and transcription played by viral RNAdependent RNA polymerase ( $\mathrm{RdRp}$ ) which is composed of three subunits i.e., polymerase basic1 (PB1), polymerase basic 2 (PB2), and polymerase acidic (PA) (Fodor 2013; Resa-Infante et al. 2011). Both PB1 and the N-terminus of PB2 form a large central cavity for catalytic mechanism of influenza-A virus (IAV) viral replication (Hengrung et al. 2015; Pflug et al. 2014; Reich et al. 2014). The priming loop of (a $\beta$-hairpin structure) PB1 thumb domain protrudes into the central cavity and supports the sugar base of the first NTP during de nova initiation (Reich et al. 2014; Appleby et al. 2015; Butcher et al. 2001; Tao et al. 2002). Moreover, primer-independent replication on the viral RNA (vRNA) template was also initiated by the priming loop (Te Velthuis et al. 2016). However, no drug exits presently in the market that blocks the entry of putative NTP and inhibits the initiation of vRNA priming loop occupying to the catalytic site of IAV RdRp. Apart from the catalytic site, IAV RdRp contains multiple sites for potential antivirus 
drug development due to its high conservation among different strains (Babar et al. 2014).

Recently, four different active sites of IAV RdRp were predicted computationally (Pagadala 2019). At present, three major classes of drugs are under development for influenza: RNA synthesis inhibitors, cap-snatching inhibitors, and inhibitors targeting protein-protein interactions between the three polymerase subunits (e.g., PA-PB1, PB1-PB2). Inhibition of IAV RNA synthesis during chain elongation is mainly inhibited by nucleoside mimicking's such as favipiravir (T-705), 2'-deoxy-2'-fluoroguanosine (Furuta et al. 2009; Tisdale et al. 1995). On the other hand, the pyrazine carboxamide derivatives (T-1105 and T-1106) show different antiviral activity on the type of host cells used. Both ribavirin and its amidine prodrug viramidine are converted to ribavirin monophosphate and acts on cellular enzyme inosine $5^{\prime}$-monophosphate dehydrogenase leading to the inhibition of vRNA synthesis (Sidwell et al. 2005; Ghanem et al. 2007). In comparison with ribavirin, cytotoxicity of viramidine is lesser which lead to further evaluation as a possible anti-influenza viral therapy. Unlike ribavirin, the influence of T-705 on cellular DNA or RNA synthesis was negative, indicating its high specificity and lesser cytotoxicity. Recently, a set of compounds that were screened targeting IAV RNA synthesis revealed that $2^{\prime} \mathrm{F}-2^{\prime}$ dNTP nucleoside analogs inhibit $\mathrm{cPol}$ and $\mathrm{vPol}$ in a completely different way (Reich et al. 2017). A combination therapy of amantadine and oseltamivir as well as favirpiravir and oseltamivir in H5N1 infected mice has shown increased in drug efficacy (Ilyushina et al. 2007). Although the combination of oseltamivir and ribavirin was shown effective, ribavirin has shown some undesirable toxic effects. Recently, combination therapy with three antiviral drugs (amantadine, ribavirin, and oseltamivir) has shown a strong effect on drug-resistant viruses including pH1N1 influenza in Korea (Kim et al. 2011). In continuation, the TCDA therapy also showed similar pharmacokinetics compared with mono-drug therapy with increased safety in immune compromised patients (Kim et al. 2011; Seo et al. 2013).

In continuation of the previous publication of known molecules of anti-influenza nucleoside analogs that were approved for the treatment of influenza viral infection and their mechanism of binding, in the present study, (Sherman et al. 2019) the compound database of $\sim 800$ nucleoside triphosphates that are commercially available was developed for molecular docking against the built homology model in three stages of replication mechanism I. In the presence of influenza-A vRNA; II. In the presence of Influenza-A vRNA with extended primer; and III. In the presence of influenza-B vRNA (Pagadala 2019). Based on the docking studies, the top ranking 11 nucleoside triphosphates that bind commonly to the catalytic site in all the three stages with higher affinities and are predicted to inhibit the IAV viral replication were chosen and their ligand protein interactions were studied further. In addition, the binding modes of 11 NTPs against IAV RdRp were predicted and their dihedral angles $\Phi_{\mathrm{T}} \mathrm{C}\left(2^{\prime}\right)-\mathrm{C}\left(1^{\prime}\right)-\mathrm{N}(1)$ -N (5)/C (6) and $\Phi_{\mathrm{P}} \mathrm{O}\left(5^{\prime}\right)-\mathrm{C}\left(1^{\prime}\right)-\mathrm{N}(1)-\mathrm{N}(5) / \mathrm{C}(6)$ that play an important role in ligand binding were analyzed. Furthermore, $25 \%\left(\mathrm{IC}_{25}\right), 50 \%\left(\mathrm{IC}_{50}\right)$, and $95 \%\left(\mathrm{EC}_{95}\right)$ effective concentrations of these 11 NTPs were calculated for IAV RdRp inhibition using trichloroacetic acid (TCA) precipitation assay (Pagadala 2019). Finally, $\operatorname{LogIC}_{50}$ of the molecules that showed inhibition was correlated with $\Delta G$ and their regression analysis $R^{2}$ was calculated.

\section{Methodology}

\section{Molecular docking}

The database of $~ 800$ nucleoside triphosphates developed from different chemical companies were used for molecular docking using the same methodology that was published earlier with the homology model of $\mathrm{A} / \mathrm{PR} / 8 / 34 / \mathrm{H} 1 \mathrm{~N} 1$ polymerase in three different stages of replication mechanism; I: In the presence of influenza-A vRNA, II. In the presence of Influenza-A vRNA with extended primer, and III. In the presence of influenza-B vRNA using MOE (Chemical computing group, Montreal, Canada) (Pagadala 2019). Initially, the ligand was optimized by energy minimization with TAFF forcefield until the energy gradient is below $0.001 \mathrm{Kcal} / \mathrm{Mol}$ (Clark et al. 1989). Totally 1000 docking poses for each ligand were generated using implicit generalized born solvation model and ligand placement method "Alpha PMI" with London dG scoring function. The initial and final scoring of the top docking poses were refined using the same London $\mathrm{dG}$ scoring function calculated with TAFF forcefield (Mackerell et al. 2004). At the end, global minimum of the ligand was predicted using the simulated annealing based on the Monte Carlo method (Metropolis et al. 1953). Finally, the affinity of the docking poses as the sum of the electrostatic, and Van der Waals energies were ranked with $\Delta G$ ( $U$ total in $\mathrm{kcal} / \mathrm{Mol})$. The reaction model cutoff between 8 and $10 \AA$ as a dielectric function with the pocket radius of $6 \AA$ was included in our docking studies. In total, the 30 docking conformations were retained as a cutoff.

\section{TCA precipitation assay}

We then investigated the effect of top ranking 11 compounds with higher MW and TPSA and bind to the catalytic site of RdRp inhibiting the incorporation of GTP into TCA-precipitable material with the A/PR/8/34 (PR8) strain (Table 1 and Fig. 1). The purified influenza virus A/PR/8/ 


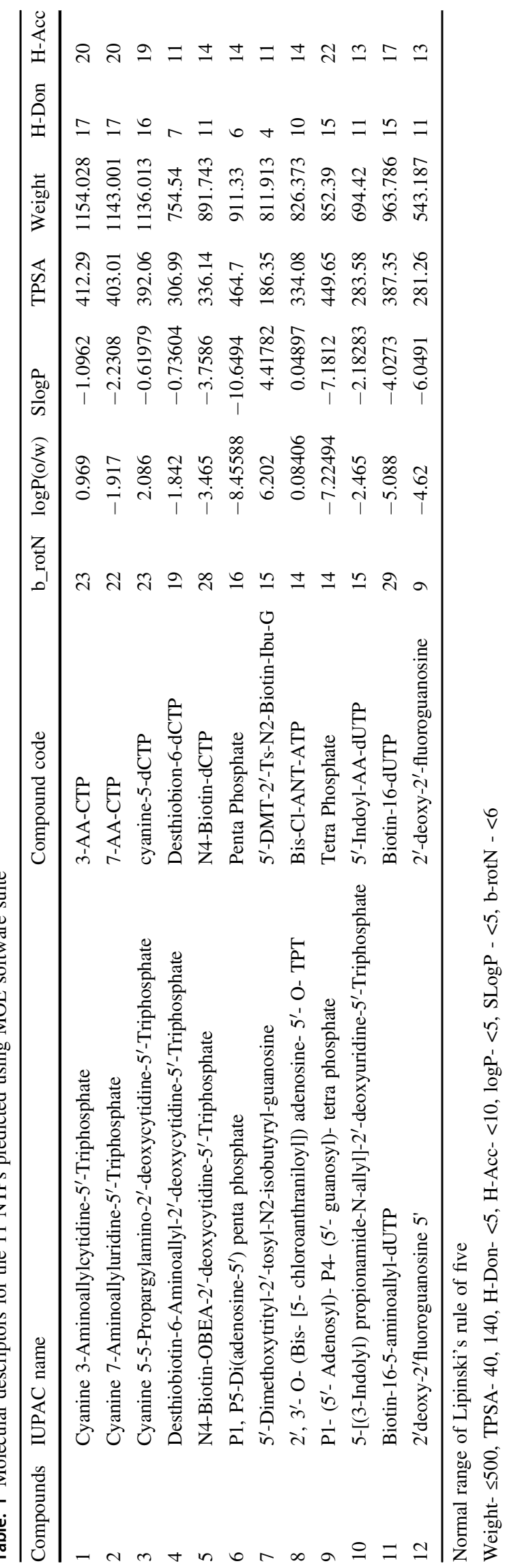

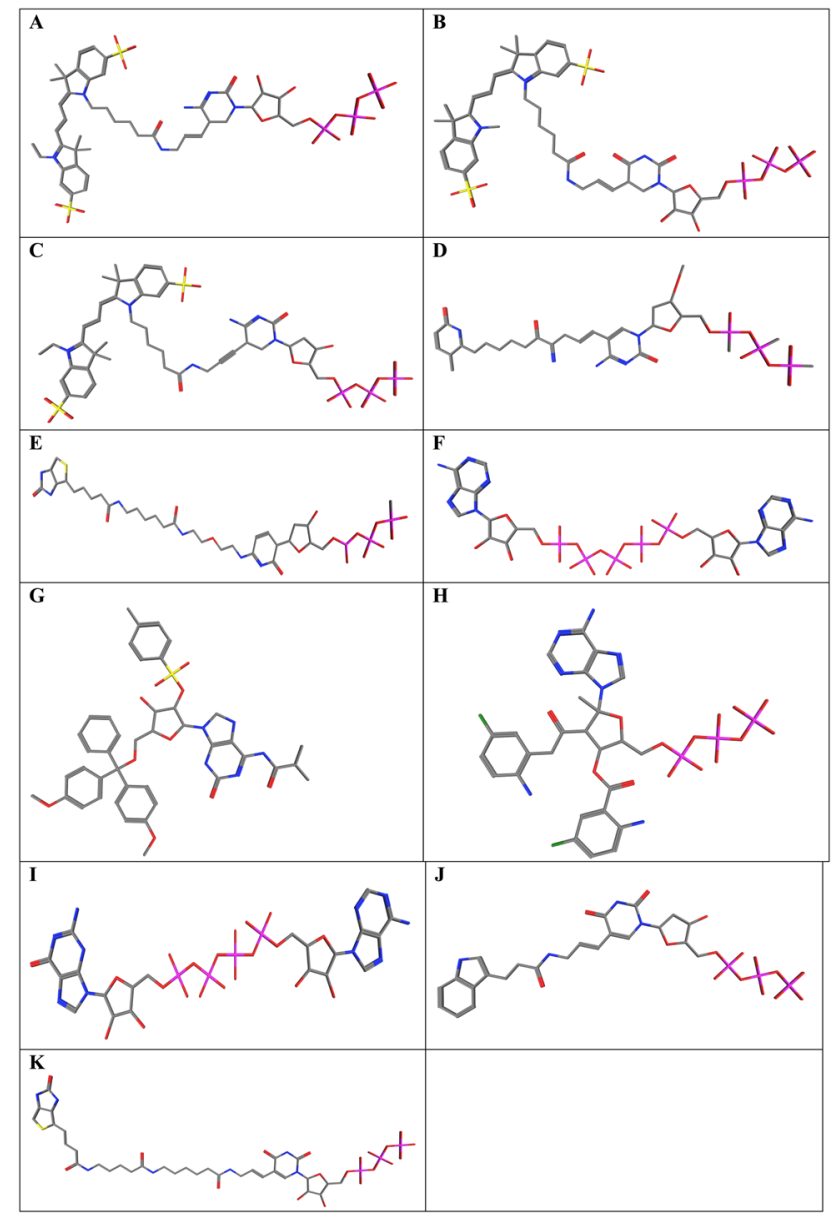

Fig. 1 Top 11 compounds 3-AA-CTP (a), 7-AA-CTP (b), cyanine-5dCTP (c), Desthiobion-6-dCTP (d), N4-Biotin-dCTP (e), Penta phosphate (f), 5'-DMT-2'-Ts-N2-Biotin-Ibu-G (g), Bis-Cl-ANT-ATP (h), Tetra phosphate (i), 5'-Indoyl-AA-dUTP (j), and Biotin-16-dUTP (k) that bind with higher affinity to the homology model catalytic site of IAV RdRp from A/PR/8/34/H1N1 strain predicted using MOE software suite

34/H1N1 obtained from Charles River (MD) was disrupted with $2.5 \%$ Triton $\mathrm{N}-101$ and diluted $1: 2$ with $0.25 \%$ Triton N-101. Disruption provided the source of influenza ribonucleoprotein (RNP) containing the IAV RdRp and template vRNA. Samples were stored on ice until use in the assay. Six serial half-log dilutions of the test drugs (twelve drugs) at concentrations of $0.001,0.01,0.1,1,10$, and $100 \mu \mathrm{M}$ and positive control 2'Deoxy-2'fluoroguanosine 5'triphosphate (high tests of $100 \mu \mathrm{M}$ ) were tested in triplicate. Each polymerase reaction contained the following: disrupted RNP, Tris- $\mathrm{HCl}, \mathrm{KCl}, \mathrm{MgCl}_{2}$, Dithiothreitol, $0.25 \%$ Triton N-101, $\left[\alpha-{ }^{32} \mathrm{P}\right]$ GTP, ATP, CTP, UTP, GTP, and Adenyl $\left(3^{\prime}-5^{\prime}\right)$ Guanosine. For testing the inhibitor, the reactions contained the inhibitor and the same was done for reactions containing the positive control. The reaction was incubated at $30^{\circ} \mathrm{C}$ for $1 \mathrm{~h}$, transferred onto glass-fiber filter plates and subsequent precipitation of nucleic acids with 
$10 \%$ TCA. The filters were then washed with 5\% TCA followed by $95 \%$ ethanol and air dried. Once the filter has dried, incorporation of $\left[\alpha^{32} \mathrm{P}\right]$ GTP was measured using a scintillation counter (Micro beta). Radioactivity was measured in a Micro beta liquid scintillation counter. Negative control reactions were prepared by omitting RNP complexes, whereas, positive control reactions for polymerase inhibitors contained the specific inhibitor 2'-deoxy-2'Fluoroguanosine $5^{\prime}$-triphosphate. Inhibition of viral polymerase activity was measured by $\mathrm{IC}_{25}, \mathrm{IC}_{50}$, and $\mathrm{IC}_{95}$ in triplicate (Pagadala 2019).

\section{Results}

\section{Binding modes of 11 NTPs against the homology model of human A/PR/8/34/H1N1 RdRp}

\section{Stage-I: In the presence of influenza-A vRNA}

In the docked complex, compound 1 binds to the catalytic site with both ribose sugar and cytidine nitrogen interacting with $\mathrm{Ser}^{494}$ and $\mathrm{Ala}^{648}$ of the priming loop through $2^{\prime}$ and backbone oxygens. The central $1 \mathrm{H}$-indole ring and sulfonyl oxygen atoms contacts with $\mathrm{Asn}^{42}$ and $\mathrm{Leu}^{45}$ of PB2-N involved in interactions with the 3 'activator strand of vRNA once it enters the polymerase active site. However, the terminal $1 \mathrm{H}$-indole ring contacts with Pro ${ }^{641}$ of PB1 priming loop through ethyl carbon. Also, the $\alpha$ and $\gamma$ phosphates contact with side chains of $\mathrm{Asn}^{536}$ and $\mathrm{Arg}^{142}$ at the end of the second tunnel (Fig. 2a). Due to its bigger size, compound 2, occupies more space towards the catalytic site showing interactions between cyanine and $\mathrm{Met}^{646}$ of the priming loop. In addition, 7-Allyl also shows contact with $\mathrm{Met}^{646 \text { and } 507}$ while the ribose sugar towards the exit channel makes contacts with $\mathrm{Asn}^{504}$, $\mathrm{Glu}^{508}$, and $\mathrm{Asn}^{537}$ of PB1 in the central region of a typical right-handed RdRp fold. The central sulfonyl at the C-terminal region contacts with $\mathrm{Asn}^{42}, \mathrm{Pro}^{43}$, and $\mathrm{Ala}^{44}$ of PB2-N through backbone atoms (Fig. 2b). In comparison, the terminal $1 \mathrm{H}$-indole ring of compound 3 shows H-Pi stack with $\mathrm{Glu}^{232}$ and $\mathrm{Arg}^{233}$ near the NTP tunnel placing the sulfonyl oxygens close to $\mathrm{Mg}^{++}$. In addition, the central $1 \mathrm{H}$-indole ring and the attached sulfonyl oxygen also interacts with $\mathrm{Met}^{646}$ and $\mathrm{Ser}^{511}$ of PB1 central domain through H-Pi and hydrogen bond. This orientation is further stabilized by additional contacts with $\mathrm{Met}^{646}$ and $\mathrm{Ala}^{648}$ of the priming loop through the central 5-5-propargylamino carbon and oxygen atoms. Other than these interactions, the ribose sugar, $\alpha$ and $\gamma$ phosphates also interacts with $\mathrm{Thr}^{493}, \mathrm{Asn}^{537}$, and $\mathrm{Asn}^{276}$ of PB1 central domain (Fig. 2c).

Furthermore, it was demonstrated that compound 4 occupies the binding pocket at the end of tunnel 2 with

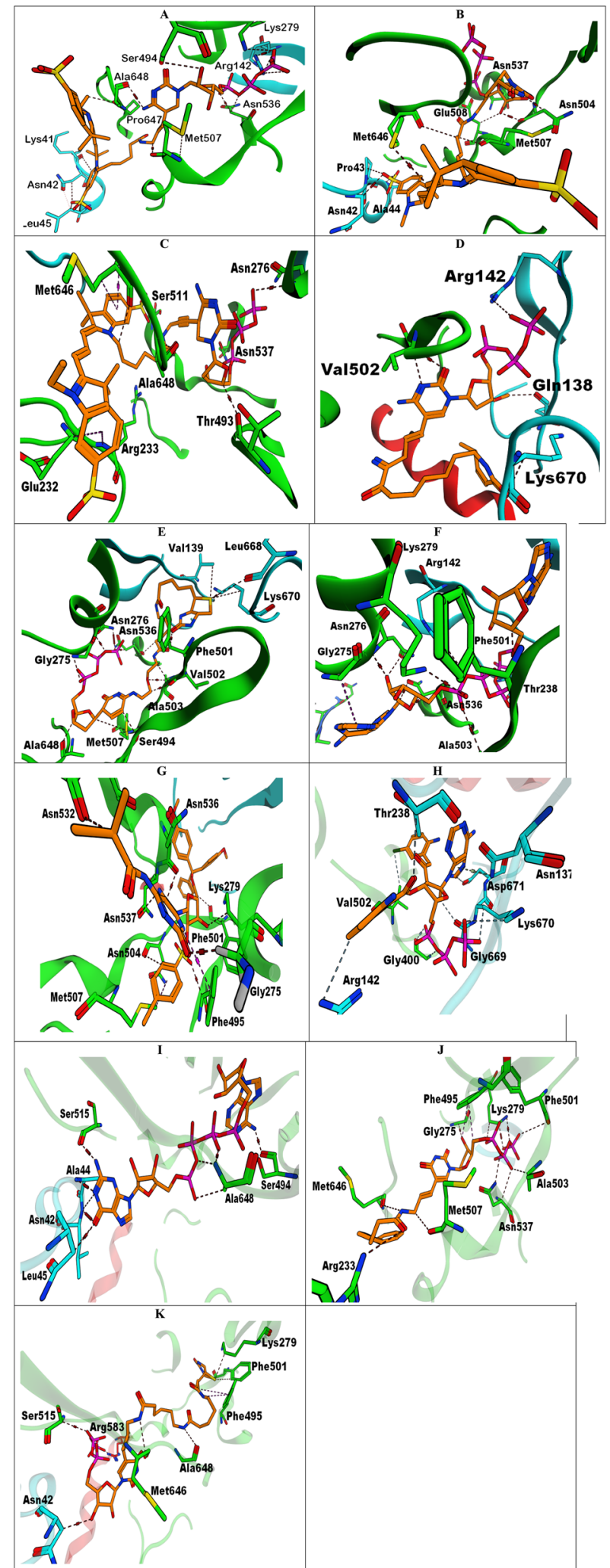

Fig. 2 Ligand protein interaction of top 11 compounds represented in codes 3-AA-CTP (a), 7-AA-CTP (b), cyanine-5-dCTP (c), Desthiobion-6-dCTP (d), N4-Biotin-dCTP (e), Penta phosphate (f), $5^{\prime}$ DMT-2'-Ts-N2-Biotin-Ibu-G (g), Bis-Cl-ANT-ATP (h), Tetra phosphate (i), 5'-Indoyl-AA-dUTP (j), and Biotin-16-dUTP (k) with the homology model of IAV RdRp from A/PR/8/34/H1N1 strain 
$\gamma$-phosphate and oxymethyl of ribose sugar makes contacts with PB2 linker $\mathrm{Asn}^{138}$ and $\mathrm{Arg}^{142}$ that leads to an Nterminal subdomain. The cytidine which is placed far away from the PB2 linker shows contacts with $\mathrm{Val}^{502}$ of PB1 central domain. This allows 6-aminoally aliphatic chain to enter deeply into the pocket forming a U-shape, exposing desthiobiotin at the other end of the molecule to interact with Lys ${ }^{670}$ of PB2 627 linker (Fig. 2d). When desthiobiotin-6-Aminoallyl was replaced with $\mathrm{N}^{4}$-biotinOBEA, compound 5 penetrates much deeper than compound 4 in the same binding site. The sulfur atom of biotin contacts with $\mathrm{Val}^{139}$, Leu ${ }^{668}$, and Lys ${ }^{670}$ of PB2-N subdomain and PB2 627 linkers, while the oxo group interact with $\mathrm{Phe}^{501}$ of PB1 central domain. In addition, the OBEA aliphatic chain also shows contacts with $\mathrm{Val}^{502}, \mathrm{Ala}^{503}$, and $\mathrm{Asn}^{536}$ through backbone carbon, and oxygen atoms. Moreover, the cytidine and ribose sugar along with $\alpha, \beta$, and $\gamma$ phosphates shows contacts with $\mathrm{Ser}^{494}, \mathrm{Met}^{507}$, $\mathrm{Ala}^{648}$, $\mathrm{Gly}^{275}, \mathrm{Asn}^{276}$, and $\mathrm{Asn}^{536}$ of PB1 central and priming loop forming more or less a circular fold. This shows that compound 5 binds with a greater number of contacts than compound 4 in the same binding site influencing both PB1 priming loop and PB2 linker domain involved in RNA catalysis (Fig. 2e).

Along with the above, compound 6 also binds to the same binding site that was identified previously with compounds 4 and 5 with one end of adenosine moiety shows $\mathrm{Pi}$ stack with Gly ${ }^{275}$ and the adenosine at the other end orients towards the PB2 627 linker domain without showing any contacts with IAV RdRp. The terminal oxygens of both the $\alpha$ and $\gamma$ phosphates show interactions with Lys $^{279}$ and $\mathrm{Ala}^{503}$ of PB1 central domain and $\mathrm{Thr}^{238}$ of PB2-N-terminal domain keeping both the domains intact. On the other hand, the ribose sugars of both the sides also show contacts with $\mathrm{Asn}^{276}, \mathrm{Asn}^{536}$, and Phe ${ }^{501}$ of PB1 central domain through carbon, and oxygen atoms (Fig. 2f). However, the benzyl ring of compound 7 shows $\mathrm{H}-\mathrm{Pi}$ stacking with $\mathrm{Me}^{507}$ with sulfonyl oxygen interacting with Phe ${ }^{495}$ of the PB1 central domain. Also, the ribose sugar interacts with $\mathrm{Lys}^{279}$ and $\mathrm{Val}^{502}$ of PB1 central domain through $2^{\prime}$ oxygen and carbon. In addition, the $5^{\prime}$-dimethoxytrityl group also contacts with $\mathrm{Val}^{502}$ through phenyl carbon. In comparison, the adenosine ring forms $\mathrm{H}-\mathrm{Pi}$ interactions with $\mathrm{Asn}^{137}$ of $\mathrm{PB} 2$ near the entry channel. In addition, the adenosine and ribose rings also to show contacts with Asp ${ }^{671}$ and Gly ${ }^{669}$ of PB2 linker domain through backbone and methyl carbons. Moreover, the attached $\alpha$ and $\gamma$ triphosphates also orient towards the linker domain showing contacts with Gly ${ }^{500}$ of PB1 central domain and Lys ${ }^{670}$ of PB2 627 linker through terminal oxygens (Fig. 2g). However, both the anthraniloyl rings of compound 8 in $2^{\prime}$ and $3^{\prime}$ shows contacts with $\mathrm{Val}^{502}$ $\operatorname{Arg}^{142}$ both PB1 and PB2 domains through terminal chlorine. Other than these contacts, oxo group of $2^{\prime}$ ribose sugars also interact with $\mathrm{Thr}^{238}$ of PB2 through a backbone carbon (Fig. 2h).

When compound 6 is replaced with guanosyl moiety limiting the number of phosphates to four allowing the compound 9 to occupy both the tunnels with a priming loop in between. The $5^{\prime}$ adenosyl group is placed in tunnel 1 orienting towards the $\mathrm{C}$-terminal domain of $\mathrm{PB} 2$ interacting with $\mathrm{Asn}^{42}, \mathrm{Ala}^{44}$, and $\mathrm{Leu}^{45}$ while $5^{\prime}$ guanosyl placed in tunnel 2 shows contacts with $\mathrm{Ser}^{494}$ near to the exit channel. In addition, the $\alpha$ and $\beta$ phosphates also contact with $\mathrm{Ala}^{648}$ of the priming loop through terminal oxygens (Fig. 2i). The propionamide- $\mathrm{N}$-allyl of compound 10 shows interactions with $\mathrm{Arg}^{233}, \mathrm{Met}^{507}$, and $\mathrm{Met}^{646}$ of the PB1 priming loop through oxo, nitro, and carbon atoms, leaving the indole ring to move freely in the catalytic site of IAV RdRp. Both the ribose sugar and $\alpha, \beta$, and $\gamma$ phosphates in tunnel 2 interacts with $\mathrm{Gly}^{275}$ and $\mathrm{Phe}^{495}$, $\mathrm{Lys}^{279}, \mathrm{Ala}^{503}, \mathrm{Asn}^{537}$, and $\mathrm{Phe}^{501}$ of PB1 through $3^{\prime}$ and terminal oxygens (Fig. 2j). Furthermore, when 5-[(3-Indolyl) propionamide-N-allyl] group is replaced with Biotin-16-5-aminoallyl allows compound 11 to bind both the tunnels showing contacts with the priming loop of the catalytic site. The biotin sulfur interacts with $\mathrm{Lys}^{279}$ and $\mathrm{Phe}^{501}$ and the aminoallyl contacts with $\mathrm{Phe}^{495} \mathrm{Met}^{646}$ and $\mathrm{Ala}^{648}$ of PB1 central domain, including the priming loop, through backbone nitrogen and carbon. Furthermore, ${ }^{\prime}$ 'ribose sugar along with $\beta$ and $\gamma$ phosphates contacts with $\mathrm{Asn}^{42}$ of PB2-N, $\mathrm{Ser}^{515}$ and $\mathrm{Arg}^{583}$ of both PB1 central domain and PA-C-terminal domain through oxygen atoms (Fig. 2k).

The bind modes of compounds 1-3 show that cyanine moiety is placed below the priming loop perpendicular to the tunnel 1 with the rest of the chain appears in parallel to the tunnel 2 almost showing a V-shape. This reveals that compounds 1 and 3 with cytidine moiety are more effective in binding to IAV RdRp than compound with uridine moiety. These compounds follow the order in binding to IAV RdRp, compound $1>$ compound $3>$ compound 2 . In comparison, the binding orientation of compounds 4-6, 9, and compounds 10-11 shows linearity in the catalytic site interacting with PB1 priming loop. In case of compounds 4 and 5 , both occupy tunnel 2 with biotin orienting towards the exit channel. However, compound 4 is close to the PB2 linker domain while the same moiety stays nearer to PB1 priming loop. Surprisingly, compound 6 with Penta phosphate completely occupies tunnel 2 with respect to compound 9 having tetra phosphate which occupies tunnel 2 partially. This may be due to the presence of guanosyl at one end in compound 10 instead of adenosyl at both the ends in compound 6. Furthermore, comparison of compounds 10 and 11 clearly indicates that 2 -deoxyuridine-5'triphosphate of both these compounds in tunnel 2 are placed quite opposite to each other with 5-[(3-Indolyl) propionamide-N-allyl] of compound 10 is placed close to PB1 
priming loop while biotin of compound 11 is placed close to PB2 linker domain. Although, both compounds 7 and 8 occupy tunnel 2 , compound 7 is placed closed to PB2 linker domain while compound 8 resides close to the PB1 priming loop.

The docking results of these compounds show that the residues $\mathrm{Asn}^{504}$ from PA; $\mathrm{Arg}^{142}, \mathrm{Met}^{227}, \mathrm{Arg}^{233}, \mathrm{Thr}^{238}$, $\mathrm{Gly}^{275}, \mathrm{Asn}^{276}, \mathrm{Lys}^{279}, \mathrm{Arg}^{287}, \mathrm{Asn}^{413}, \mathrm{Met}^{414}, \mathrm{Ser}^{494}$, $\mathrm{Phe}^{495}$, Phe ${ }^{496}, \mathrm{Gly}^{500}, \mathrm{Phe}^{501}, \mathrm{Val}^{502}, \mathrm{Ala}^{503}, \mathrm{Met}^{507}$, $\mathrm{Gly}^{513}, \mathrm{Ser}^{515}, \mathrm{Asn}^{536}, \mathrm{Met}^{646}, \mathrm{Ala}^{648}$, from PB1 and $\mathrm{Asn}^{42}$, $\mathrm{Ala}^{44}, \mathrm{Leu}^{45}$, $\mathrm{Lys}^{140}{ }^{14 \mathrm{Arg}^{141}}{ }^{\text {, Lys }}{ }^{670}$, and Leu ${ }^{668}$ from PB2 act as common pharmacophores for these compounds preventing the activity of $\mathrm{A} / \mathrm{PR} / 8 / 34 / \mathrm{H} 1 \mathrm{~N} 1$ influenza-A polymerase.

\section{Stage-II: In the presence of Influenza-A vRNA with extended primer}

In the presence of extended primer from $\phi 6$ polymerase, the $5^{\prime}$ triphosphate of compound 1 interacts with $\mathrm{Asn}^{513}, \mathrm{Arg}^{38}$, and C11 of PA-C, PB2, and vRNA activator strand at the $3^{\prime}$ end. Furthermore, the ribose sugar also interacts with the $\mathrm{Gln}^{127}$ and $\mathrm{Met}^{227}$ of PB1 and Lys ${ }^{41}$ of PB2-N-ter through oxygen atoms $\mathrm{O}_{66}, \mathrm{O}_{91}$, and $\mathrm{O}_{95}$. The $\mathrm{C}_{30}$ indole ring forms $\mathrm{H}-\mathrm{Pi}$ stack with $\mathrm{Arg}^{239}$ of motif-F3 and the terminal sulfonyl orient towards Phe $\mathrm{P}^{412}$ and $\mathrm{Asn}^{413}$ of PB1 motif-III. The 3allyl group also shows contact with $\mathrm{Met}^{409}$ of Motif-B Methionine rich loop through backbone carbon $\mathrm{C}_{38}$ (Fig. $3 a)$. Due to its bigger size, compound 2 occupies more space in the tunnel 2 just above the priming loop of PB1. The oxygen atoms of uridine and ribose sugar shows contact with Phe ${ }^{501}$ and Asn ${ }^{536}$ of PB1 and Lys ${ }^{140}$ of PB2 at the end of the tunnel 2. In addition, the 7-Allyl group also shows contact with $\mathrm{Asn}^{536}$ and $\mathrm{Asn}^{276}$ of PB1 catalytic site above the priming loop. However, triphosphate orients towards the exit channel and makes contact with the positive changed lys ${ }^{140}$ of PB2-N and Lys ${ }^{670}$ of PB2 627 domains. Both the sulfonyl groups attached to $1 \mathrm{H}$-indole ring contacts with $\mathrm{Ser}^{494}$ of PB1 central, Ala ${ }^{648}$ and His ${ }^{649}$ of PB1 priming loop and $\mathrm{Arg}^{216}$ of PB2-N laying almost in parallel to the priming loop (Fig. 3b). The cyanine group of compound 3 also lies in almost parallel to the priming loop of PB1 with a sulfonyl group attached at the two ends of the indole interacts with $\mathrm{Met}^{507}$ and $\mathrm{Asn}^{536}$ of PB1 central and $\mathrm{Ala}^{648}$ of the PB1 priming loop. The 5-5-propargylamino and $2^{\prime}$-deoxycytidine interacts with $\mathrm{Asn}^{536}$ and Phe ${ }^{496}$ while the terminal phosphates interaction with $\mathrm{Lys}^{279}$, $\mathrm{Val}^{502}$, and $\mathrm{Val}^{503}$ of PB1 central domain at the end of the tunnel 2 (Fig. $3 \mathrm{c})$. The $\gamma$-phosphate of compound 4 penetrates deeper at the end of the tunnel 2 showing contacts with $\mathrm{Asn}^{16}$ and $\mathrm{Ser}^{594}$ of both PB1 central and PA-C through terminal oxygens. Both cytidine and desthiobiotin rings including the aminoallyl contacts with $\mathrm{Gly}^{500}$ of PB1 central, Gln ${ }^{138}$ and $\mathrm{Arg}^{142}$ of PB2-N through oxo and nitro groups (Fig. 3d). Replacing desthiobiotin-6-Aminoallyl with N4-BiotinOBEA allows compound 5 to also bind at the end of the tunnel 2 forming a circular structure. The terminal biotin contacts with Lys $^{279}$ and Lys ${ }^{140}$ of both PB1 Central and PB2-N, while the cytidine interacts with Asn ${ }^{16}$ of PB1-N through oxo and nitrogen atoms. In addition, the $\beta$ and $\gamma$ triphosphate interacts with $\mathrm{Ile}^{535}, \mathrm{Asp}^{538}$ of PB1 central, and $\mathrm{Thr}^{238}$ of PB2-N through terminal oxygens (Fig. 3e).

In comparison with compounds 4 and 5, compound 6 with two ends of adenosine interaction with $\mathrm{Met}^{507}$ of PB1 central and $\mathrm{Met}^{645}$ of PB2 627 domains. The attached furanosyls also shows contacts with $\mathrm{Asn}^{537}$ and Gly ${ }^{500}$ of PB1 domains through $2^{\prime}$ and $3^{\prime}$ oxygens. In addition, the $\alpha, \beta$, and $\varepsilon$ triphosphates in-between shows contact with $\mathrm{Asn}^{276}$, $\mathrm{Asn}^{536}$, and Pro ${ }^{219}$ of PB1 central and PB2-N with terminal oxygens (Fig. 3f). However, the sulfonyl group of compound 7 is placed just below to the tip of the priming loop interacting with Gly ${ }^{650}$ of PB1 priming loop. The 5'-dimethoxytrityl group also makes contact with $\mathrm{Arg}^{126}$ through $\mathrm{H}$ $\mathrm{Pi}$ stacking along with $\mathrm{Met}^{646}$ of PB1 and $\mathrm{Ly}^{41}$ of PB2-N near to the entry channel of tunnel 1 . The N2-isobutyrylguanosine protrudes towards NTP tunnel showing no contacts with the viral enzyme (Fig. $3 \mathrm{~g}$ ). In comparison, the compound 8 with 5-chloroanthraniloyl groups attached to $2^{\prime}$ and $3^{\prime}$ positions of ribose sugar make $\mathrm{H}-\mathrm{Pi}$ interactions with Lys $^{140}$ and $\mathrm{Arg}^{142}$ of PB2-N at the end of the second tunnel. In addition, the adenosine and triphosphate groups attached to $1^{\prime}$ and $4^{\prime}$ positions also show contacts with $\operatorname{Gln}^{138}$ and $\mathrm{Thr}^{238}$ of PB2-N far away from the linker domain through $\mathrm{H}-\mathrm{Pi}$ and weak hydrogen bonds (Fig. 3h). However, the two ends of adenosine and guanosyl moieties of compound 9 show H-Pi stack with $\mathrm{Met}^{507}$ of PB1 central, $\mathrm{Thr}^{238}$ of PB2-N, and Lys ${ }^{670}$ of PB2 627 domains, while the attached furanosyls shows contact with $\mathrm{Val}^{502}, \mathrm{Asn}^{504}, \mathrm{Met}^{507}$ of $\mathrm{PB} 1$, and Gly ${ }^{669}$ of PB2 627 domains, respectively. In addition, the $\alpha$ and $\gamma$ phosphates also show contact with $\mathrm{Lys}^{279}, \mathrm{Asn}^{504}, \mathrm{Asn}^{536}$, and $\mathrm{Asp}^{538}$ of PB1 central through terminal oxygens. In addition, adenine also interacts with $\mathrm{Ala}^{648}$ of the priming loop through the amino group (Fig. 3i). The indole and uridine groups of compound 10 forms $\mathrm{H}-\mathrm{Pi}$ stacking with $\mathrm{Ala}^{139}$ and $\mathrm{Arg}^{126}$ of PB1 central. The propionamide-N-allyl shows contacts with the backbone atoms of $\mathrm{Gly}^{125}$ and $\mathrm{Gln}^{39}$ of both PB1 central and PB2-N through backbone nitrogen. In addition, both the sugar, and the base which occupies in tunnel 1 contacts with Lys ${ }^{229}$, $\mathrm{Asp}^{230}$, and $\mathrm{Ala}^{231}$ of PB1 central and Lys ${ }^{41}$ of PB2-N with both stronger and weaker hydrogen bonds. The attached $5^{\prime}$ triphosphate also shows contacts with $\mathrm{Ala}^{231}$ of $\mathrm{PB} 1$ central and with $\mathrm{Lys}^{41}$ and $\mathrm{Asn}^{42}$ of PB2-N domain (Fig. 2j). Furthermore, compound 11 also binds similar to compound 10 in perpendicular to the catalytic site. Biotin-16-5- 
Fig. 3 Ligand protein interaction of top 11 compounds represented in codes 3-AA-CTP (a), 7-AA-CTP (b), cyanine-5dCTP (c), Desthiobion-6-dCTP (d), N4-Biotin-dCTP (e) Penta phosphate (f), $5^{\prime}$-DMT-2'-TsN2-Biotin-Ibu-G (g), Bis-ClANT-ATP (h), Tetra phosphate (i), 5'-Indoyl-AA-dUTP (j), and Biotin-16-dUTP (k) with the homology model of IAV RdRp from $\mathrm{A} / \mathrm{PR} / 8 / 34 / \mathrm{H} 1 \mathrm{~N} 1$ strain in the presence of Flu A Primer

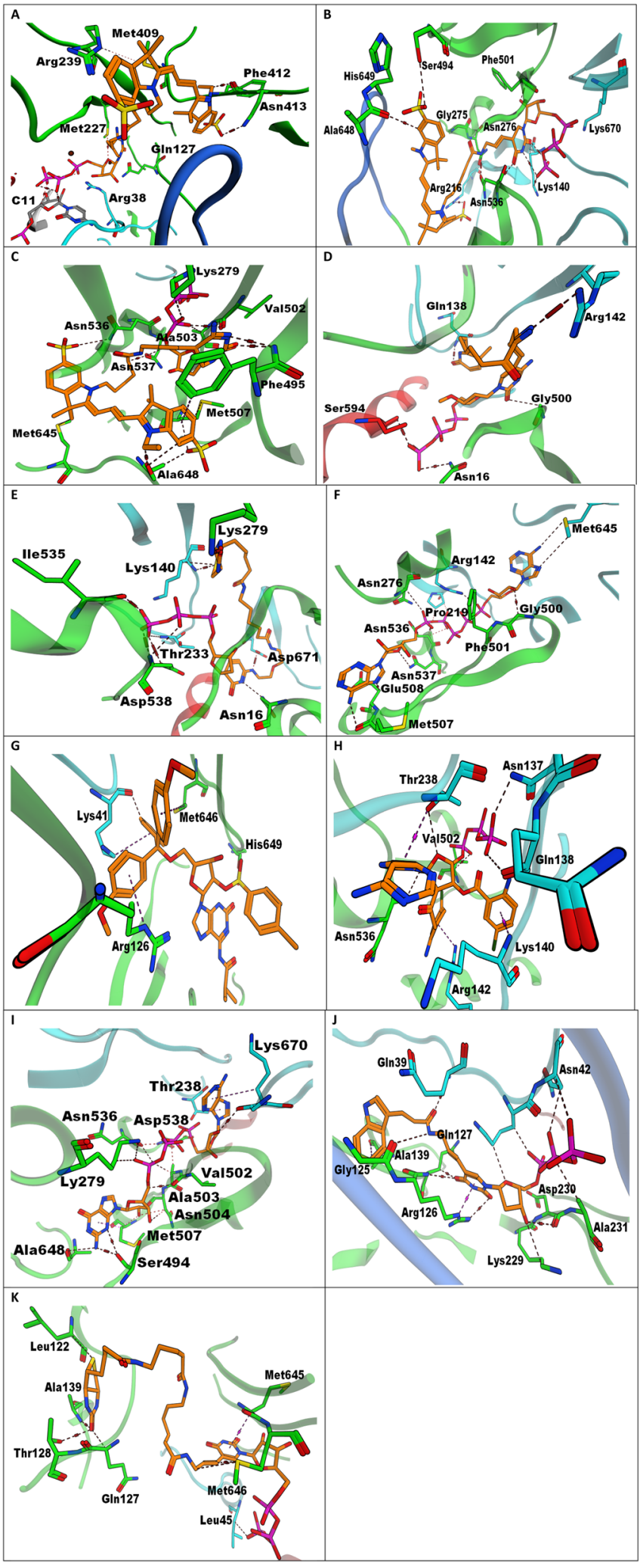

the catalytic site with triphosphate projecting into the entry channel (Fig. 3k).

The binding orientations of these compounds reveal that compound 1 occupy tunnel 1 while compounds 2 and 3 aminoallyl interacts with $\mathrm{Leu}^{122}, \mathrm{Thr}^{128}$ and $\mathrm{Ala}^{139}$ and $\mathrm{Met}^{646}$ of PB1 central while $2^{\prime}$-deoxyuridine shows H-Pi stacking with $\mathrm{Met}^{645}$ of the priming loop. This binding clearly shows that Biotin-16-5-aminoallyl orient away from 
occupied tunnel 2 above the priming loop with cyanine moiety placing in perpendicular to the tunnels 1 and 2 forming a V-shape. This proves that compound with cytidine base is more effective against polymerase inhibition than compound with uridine base. The order of compounds inhibiting RdRp is as follows, compound $3>$ compound $2>$ compound 1. Surprisingly, compound 4 also forms a Vshape while compound 5 shows circular form binding close to the PB2 linker domain with triphosphates is on the opposite sides. In comparison, the binding orientation of compounds 6 and 9 shows linearity above the catalytic site in the tunnel 2 interacting with a priming loop of PB1. The other linear shape compounds 10 and 11 binds away from tunnel 1 with $2^{\prime}$-deoxyuridine-5'-triphosphate placing in tunnel 1 close to NTP tunnel. However, compound 8 mostly occupies tunnel 2 near to the PB2 linker domain. Compared with dicyclic group of compound 8 , a tricyclic group of compound 7 binds in tunnel 1 below the PB1 priming loop with $5^{\prime}$-dimethoxytrityl orients towards the $3^{\prime}$ end of the primer and 2'-tosyl-N2-isobutyryl-guanosine towards motif$\mathrm{B}$ involved in stabilizing the base pair between the incoming NTP and the primer template.

These docking studies also show that the residues $\operatorname{Arg}^{126}$, $\mathrm{Lys}^{279}, \mathrm{Gly}^{410}, \mathrm{Phe}^{495}, \mathrm{Phe}^{501}, \mathrm{Val}^{502}, \mathrm{Asn}^{532}, \mathrm{Asn}^{536}$, $\mathrm{Met}^{646}$, and $\mathrm{Ala}^{652}$ from PB1; $\mathrm{Asn}^{41}, \mathrm{Asn}^{137}$, $\mathrm{Lys}^{140}$, and $\mathrm{Arg}^{142}$ from PB2 act as common pharmacophores of these compounds preventing the activity of $\mathrm{A} / \mathrm{PR} / 8 / 34 / \mathrm{H} 1 \mathrm{~N} 1$ influenza-A polymerase in the presence of capped primer.

\section{Stage-III: In the presence of influenza-B vRNA}

When influenza-A vRNA was replaced with influenza-B vRNA, both the nitrogenous base and the ribose sugar of compound 1 interacts with $\mathrm{Asn}^{504}$ and $\mathrm{Asn}^{537}$ through $\mathrm{C}_{60}$ and $\mathrm{O}_{91}$ atoms. The $5^{\prime}$ triphosphate contacts with $\mathrm{Asn}^{276}$ and Lys $^{279}$ at the exit channel of the RdRp through terminal oxygens. The $1 \mathrm{H}$-indole interacts with positively charged $\mathrm{Lys}^{576}$ and $\mathrm{Arg}^{583}$ of PB1 central domain through sulfonyl oxygens $124,125 \& 133$. In addition, the cytidine ring also forms, contacts with $\mathrm{Arg}^{233}$ and $\mathrm{Ala}^{648}$ at the NTP tunnel and PB1 priming loop though terminal oxygen and amino nitrogen (Fig. 4a). Also, the uridine base $\mathrm{O}_{100}$ in compound 2 shows contact with $\mathrm{Ala}^{648}$ at the tip of the priming loop while the $3^{\prime}$ carbon of ribose sugar along with triphosphate forms a Uturn in tunnel 2 interacting with Gly ${ }^{275}$ and $\mathrm{Asn}^{537}$ of PB1 central. The terminal $1 \mathrm{H}$-indole ring points towards the $3^{\prime}$ activation strand of the vRNA interacting with $\mathrm{Asn}^{42}$ and $\mathrm{Pro}^{43}$ of PB2-N through sulfonyl oxygens. In addition, the 7-Aminoally also shows contacts with $\mathrm{Pro}^{647}$ and $\mathrm{Met}^{646}$ of the PB1 priming loop (Fig. 4b). The compound 3 cyanine lies below the PB1 priming loop with central $1 \mathrm{H}$-indole ring interacting with $\mathrm{Met}^{645}$ and $\mathrm{Met}^{646}$ of PB1 priming loop while the terminal $\mathrm{IH}$-indole ring contacts with positively charged $\mathrm{Ly}^{235}$ and $\mathrm{Arg}^{239}$ of PB2 222-246 loop through sulfonyl oxygens. The 5-5-propargylamino lies perpendicular to the priming loop showing contacts with $\mathrm{Met}^{507}$, $\mathrm{Glu}^{508}$, and $\mathrm{Met}^{645}$ of PB1 central domain. This orientation of $2^{\prime}$-deoxycytidine- $5^{\prime}$-triphosphate towards the exit channel allows to interact with $\mathrm{Gly}^{275}$, $\mathrm{Asn}^{276}$, and $\mathrm{Asn}^{536}$ of PB1 and $\mathrm{Arg}^{142}$ of PB2 central (Fig. 4c). The $\alpha$ and $\beta$ phosphates of compound 4 contacts with Gly ${ }^{275}$ and $\mathrm{Asn}^{537}$ and the attached ribofuranosyl interacts with $\mathrm{Val}^{502}$ and $\mathrm{Asn}^{536}$ of PB1 central. Moreover, both the oxo and nitro group of cytidine base also shows contacts with $\mathrm{Phe}^{501}$ and $\mathrm{Asn}^{536}$ of PB1 central and $\mathrm{Pro}^{219}$ of PB2-N2 at the end of the tunnel 2 through H-Pi interaction. In addition, the desthiobiotin also shows interaction with Lys ${ }^{140}$ of the PB2-N through oxo group (Fig. 4d). The compound 5 also binds at the end of the tunnel 2 with the terminal ends of biotin and cytidine contacts with $\mathrm{Lys}^{279}$ of PB2-N and $\mathrm{Ala}^{643}$ of the priming loop through oxo groups. In addition, cytidine and furan contacts with $\mathrm{Phe}^{505}$, $\mathrm{Glu}^{505}$, and $\mathrm{Ala}^{648}$ through nitrogen and $2^{\prime}$ carbon while the $\alpha$ and $\gamma$ triphosphate interacts with $\mathrm{Gly}^{275}$ and $\mathrm{Asn}^{537}$ through terminal oxygens (Fig. 4e).

In comparison with compounds 4 and 5, compound 6 occupies both tunnel 1 and tunnel 2 almost in parallel to the catalytic site of RdRp. One end of adenosine moiety contacts along with the attached ribose sugar shows interactions with $\mathrm{Val}^{644}$, $\mathrm{Met}^{645}$, and $\mathrm{Met}^{646}$ of PB1 priming loop while the other end of adenosine orients towards the exit channel contacting with $\mathrm{Ala}^{503}$ of the PB1 central. The phosphate groups in between also interacting with $\mathrm{Glu}^{508}$ of the PB1 along with $\mathrm{Pro}^{647}$ and $\mathrm{Ala}^{648}$ of the priming loop (Fig. 4f). However, compound 7 is placed below the exit channel with $2^{\prime}$-tosyl showing contacts with $\mathrm{Ala}^{503}$ and $\mathrm{Asn}^{504}$ and the ribose sugar interacting with $\mathrm{Lys}^{279}$ and $\mathrm{Phe}^{495}$ with another two hydrogen bonds. The 5'-dimethoxytrityl group also makes contact with $\mathrm{Ile}^{535}$ and $\mathrm{Asn}^{536}$ below the exit channel Fig. 4g. Similarly, the 5-chloroanthraniloyl makes contact with $\mathrm{Asn}^{536}$ of PB1 below the exit channel of RdRp. In addition, the adenosine and triphosphate groups also show contacts with $\mathrm{Ser}^{601}$ of PA-C, Gly ${ }^{500}$ of PB1, and Gly ${ }^{669}$ of PB2 627 linker (Fig. 4h). In comparison with compound 6, the compound 9 with adenosyl at one end orients towards the priming loop of PB1 while the other end with guanosyl orients very close to Leu ${ }^{668}$ of PB2 linker domain. The 2'ribose sugar of guanosyl shows contacts with $\operatorname{Arg}^{142}$ of PB2-N2 subdomain, while the 2'ribose sugar of adenosyl contacts with Gly ${ }^{275}$ of PB1 at the exit channel. In addition, the phosphate groups also show contacts with $\mathrm{Lys}^{279}, \mathrm{Val}^{502}$, and $\mathrm{Ala}^{503}$ of PB1 central fold through terminal oxygens (Fig. 4i). The indole and propionamide-Nallyl of 5-[(3-Indolyl) propionamide-N-allyl] of compound 10 contacts with $\mathrm{Glu}^{508}$ and $\mathrm{Thr}^{493}$ of PB1 central while uridine base interacts with $\mathrm{Ala}^{648}$ of the priming loop. In 
Fig. 4 Ligand protein interaction of top 11 compounds

represented in codes 3-AA-CTP

(a), 7-AA-CTP (b), cyanine-5-

dCTP (c), Desthiobion-6-dCTP

(d), N4-Biotin-dCTP (e), Penta

phosphate (f), 5'-DMT-2'-Ts-

N2-Biotin-Ibu-G (g), Bis-Cl-

ANT-ATP (h), Tetra phosphate

(i), 5'-Indoyl-AA-dUTP (j), and

Biotin-16-dUTP (k) with the

homology model of IAV RdRp

from $\mathrm{A} / \mathrm{PR} / 8 / 34 / \mathrm{H} 1 \mathrm{~N} 1$ strain in

the presence of Flu B vRNA

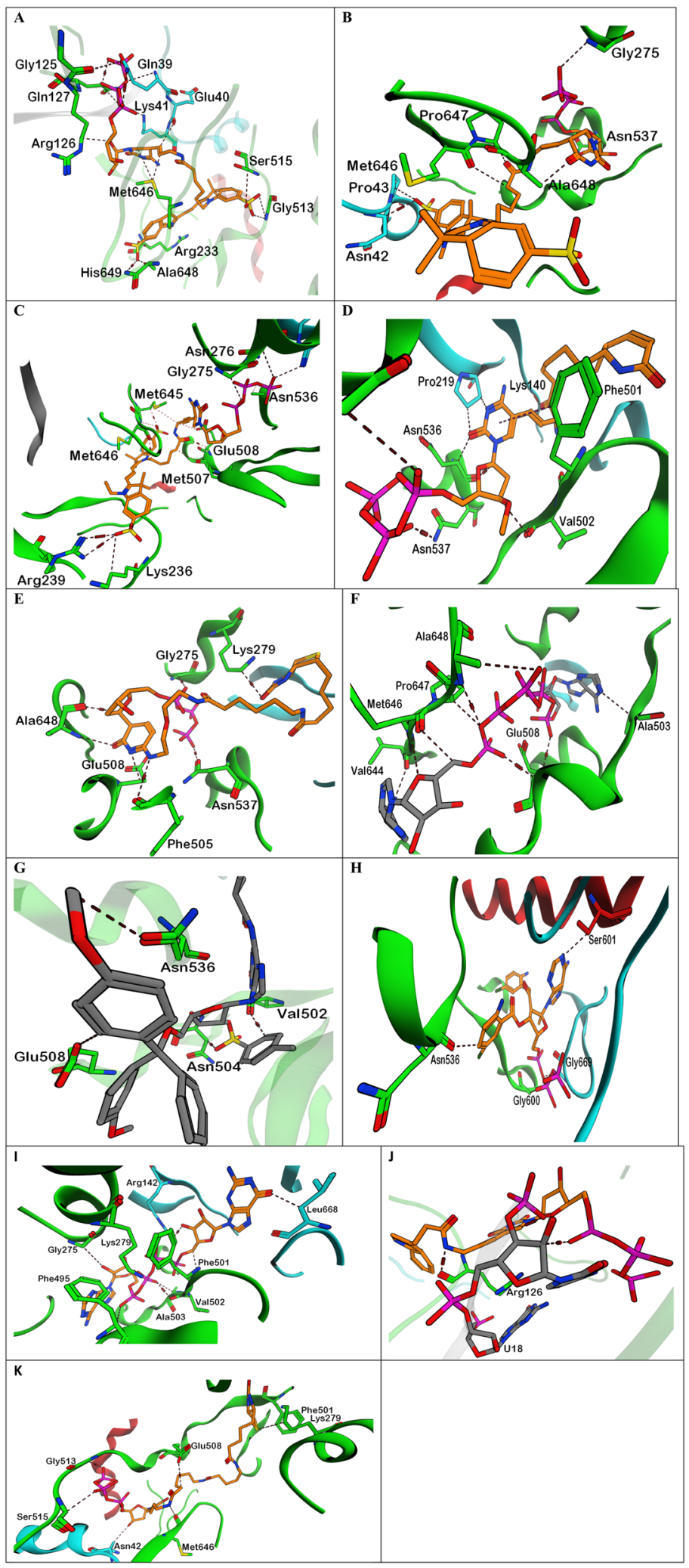

addition, the $2^{\prime}$-deoxyuridine which occupies in tunnel 1 shows contacts with Pro ${ }^{510}$ of PB1 central and the attached $5^{\prime}$-triphosphate shows contacts with $\mathrm{Ser}^{515}$ of PB1, $\mathrm{Pro}^{43}$,
$\mathrm{Ala}^{44}$, and $\mathrm{Asn}^{42}$ of PB2_C domain (Fig. 4j). However, compound 11 interacts with $\mathrm{Lys}^{279}$, $\mathrm{Phe}^{501}, \mathrm{Glu}^{508}$, and $\mathrm{Gly}^{125}$ of PB1 and $\mathrm{Met}^{646}$ of the priming loop while 
2'-deoxyuridine-5' -triphosphate shows contacts with $\mathrm{Arg}^{233}$, $\mathrm{Gly}^{513}$, and $\mathrm{Ser}^{515}$ of PB1 and $\mathrm{Asn}^{42}$ of PB2 at the Cterminal end of RdRP (Fig. 4k).

The predicted V-shape orientations for compounds 1, 2, and 3 shows that cyanine moiety is placed almost in parallel to the PB1 priming loop while the rest of the molecule projects towards the exit channel in parallel to the catalytic site of RdRp polymerase. This reveals that compounds 1 and 3 with cytidine moiety binds with higher affinity compared with compound with uridine moiety. These compounds inhibiting RdRp are as follows, compound $1>$ compound $3>$ compound 2 . The orientation of binding shows that desthiobiotin-6-aminoallyl appears in parallel to the catalytic site of RdRp in tunnel 2 with triphosphate orientation towards the priming loop while the orientation of compound 5 appears in circular with triphosphate pointing towards the exit channel. In comparison, the binding orientations of compounds 6 and 9 shows linearity above and below the catalytic site. In these orientations, it was carefully observed that the phosphate groups of compound 6 primarily interact with the priming loop which is not seen with compound 9. The other linear shape compounds 10 and 11 binds below the priming loop occupying both tunnels 1 and 2 with triphosphates towards the entry channel. However, compounds 7 and 8 mostly occupy tunnel 2 nearer to the PB2 linker domain. The predicted orientation shows that bulky groups of 5'-dimethoxytrityl and 5-chloroanthraniloyl orients towards the catalytic site while compound 7 (isobutyryl-guanosine) and compound 8 (adenosine-5'-O-triphosphate) orients towards the exit channel and the linker domain. These two binding conformations clearly indicate that compound 8 is far away from the catalytic site and PB2 linker domain compared with compound 7 which is close to the catalytic site interacting with PB1 priming loop.

The docking results of these compounds show that the residues $\mathrm{Arg}^{125}, \mathrm{Glu}^{126}, \mathrm{Ala}^{651}$, and $\mathrm{Ser}^{652}$ from PA; $\mathrm{Thr}^{39}$, $\mathrm{Ala}^{231}, \mathrm{Ala}^{503}, \mathrm{Pro}^{510}$, and $\mathrm{Met}^{646}$ from PB1 and $\mathrm{Arg}^{17}$, $\mathrm{Glu}^{18}, \mathrm{Gln}^{39}$, and $\mathrm{Lys}^{41}$ from PB2 act as common pharmacophores of these compounds preventing the activity of $\mathrm{A} / \mathrm{PR} / 8 / 34 / \mathrm{H} 1 \mathrm{~N} 1$ influenza-A polymerase after the translocation of $3^{\prime}$ end of the vRNA into the catalytic site.

\section{The activity of the compounds against IAV replication using TCA precipitation assay}

Further experimental studies using TCA precipitation assay showed that compound 1 inhibited polymerase activity with an $\mathrm{IC}_{25}$ an $\mathrm{IC}_{50}$ of 0.03 and $1.69 \mu \mathrm{M}$ with higher rates of inhibition between 0.1 and $1 \mu \mathrm{M}$ compared with other concentrations used in the study (Fig. 5a). Compounds 2 (Figs. 5b) and 3 (Fig. 5c) weakly inhibited polymerase activities, having an $\mathrm{IC}_{25}$ and $\mathrm{IC}_{50}$ of 0.8 and 3.58 and 0.6 and $2.13 \mu \mathrm{M}$, whereas compounds 4 (Fig. 5d) and 8 (Fig. 5h) had a significant effect on Flu A polymerase activity with an $\mathrm{IC}_{25}$ and $\mathrm{IC}_{50}$ of 0.06 and 0.21 and 0.27 and $0.68 \mu \mathrm{M}$, respectively. However, compounds 5 (Fig. 5e) and 6 (Fig. 5f) had a very large effect on polymerase inhibition with an $\mathrm{IC}_{25}$ and $\mathrm{IC}_{50}$ of 0.02 and 0.09 and 0.03 and $0.09 \mu \mathrm{M}$. Thus, compounds 5 and 6 displays broadspectrum antiviral activity against Flu $\mathrm{A}$ virus with $\mathrm{IC}_{50}$ at the same concentration of $0.09 \mu \mathrm{M} .2^{\prime}$-deoxy-2'-fluoroguanosine $5^{\prime}$-triphosphate, a known inhibitor of Flu A polymerase exhibited a $50 \%\left(\mathrm{IC}_{50}\right)$ effective concentration at $1.2 \mu \mathrm{M}$. For compounds 2 and 3, the higher rate of polymerase inhibition was seen between 0.1 and $1 \mu \mathrm{M}$ while for the compounds 4,5 , and 7 the higher rate of polymerase inhibition was seen at the lesser concentration between 0.1 and $1 \mu \mathrm{M}$. However, for compound 6 , the higher rate of polymerase inhibition was seen completely at a very low concentration between 0.01 and $0.1 \mu \mathrm{M}$ compared with all other compounds used in the study. Moreover, all these eight NTPs show $95 \%\left(\mathrm{EC}_{95}\right)$ of viral polymerase inhibition greater than $100 \mu \mathrm{M}$. However, no significant effect of compounds $8-11$ (Fig. 5i-k) was observed against the activity of the influenza polymerase even at higher concentration of $100 \mu \mathrm{M}$.

A regression analysis of $\log \mathrm{IC}_{50}$ with the docked scores, $\Delta G$ for the compounds 1-7 including the positive control $2^{\prime}$ deoxy-2'-fluoroguanosine $5^{\prime}$-triphosphate in the presence of influenza-A vRNA (Stage-I) (Table 2), influenza-A vRNA with extended primer (Stage-II) (Table 3) and influenza-B vRNA (Stage-III) (Table 4), were carried out and their scatter plots was drawn. It was found that these compounds showed a positive correlation with an $R^{2}$ value of 0.21 , and 0.20 in Stage-I and Stage-III and a negative correlation of 0.001 in Stage-II. However, the best correlation was obtained with an $R^{2}$ value of 0.73 and 0.57 (Fig. 6) when $\Delta G$ for the positive control $2^{\prime}$-deoxy-2'-fluoroguanosine $5^{\prime}$ triphosphate were removed from Stage-I and Stage-III. However, no change in $R^{2}$ value was seen in Stage-II even after removing the $\Delta G$ for the positive control.

\section{Discussion}

Here we report the identification of 11 NTPs that binds to the homology model of $\mathrm{A} / \mathrm{PR} / 8 / 34 / \mathrm{H} 1 \mathrm{~N} 1$ influenza virus RNA polymerase with higher affinities. The docking studies in the Stage-I and III confirm that all these linear and Vshaped compounds 1-3 except compound 11 with influenza-A vRNA commonly binds to the catalytic site around the priming loop of PB1 domain. However, compounds 10 and 11 occupies the catalytic site while the rest of the molecules bind to tunnel 2 in the presence of capped primer from $\phi 6$ polymerase. Both the cyclic compounds 7 
Fig. 5 Inhibitory concentration values $\left(\mathrm{IC}_{50}\right)$ of the IAV RdRp from $\mathrm{A} / \mathrm{PR} / 8 / 34 / \mathrm{H} 1 \mathrm{~N} 1$ strain with the top 11 compounds represented in codes 3-AA-CTP (a), 7-AA-CTP (b), cyanine-5dCTP (c), Desthiobion-6-dCTP (d), N4-Biotin-dCTP (e), Penta phosphate (f), 5'-DMT-2'-TsN2-Biotin-Ibu-G (g), Bis-ClANT-ATP (h), Tetra phosphate (i), 5'-Indoyl-AA-dUTP (j), and Biotin-16-dUTP (k) using TCA precipitation assay.

Concentration of the drug $(\mu \mathrm{M})$ is shown in $X$-axis and \% of virus control is shown in $Y$-axis
A

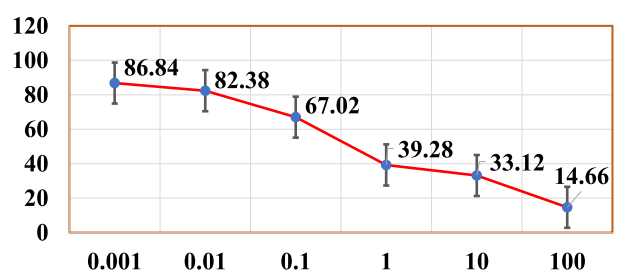

C

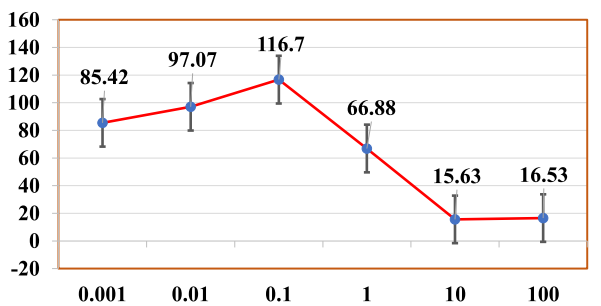

E

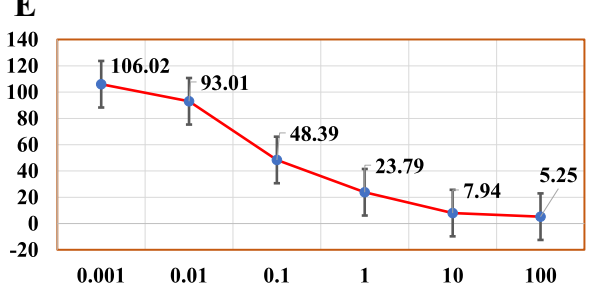

G

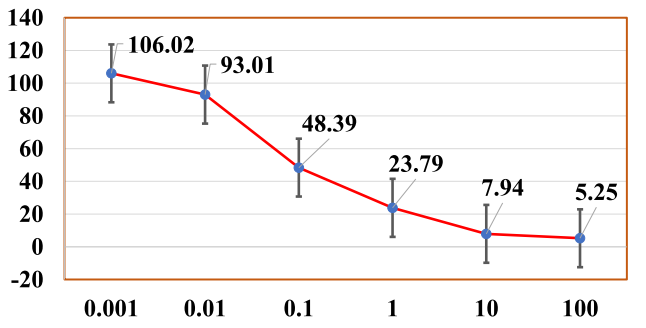

I

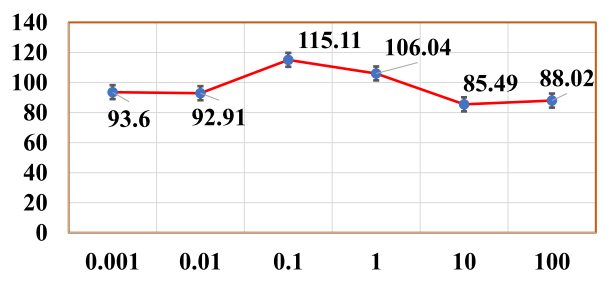

B

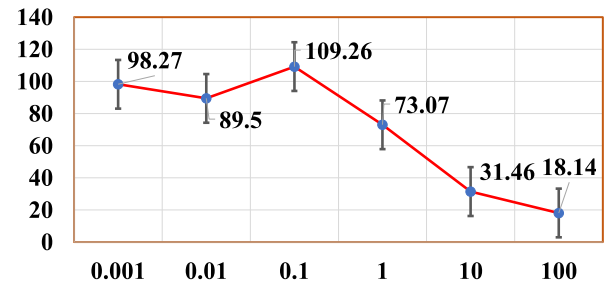

D

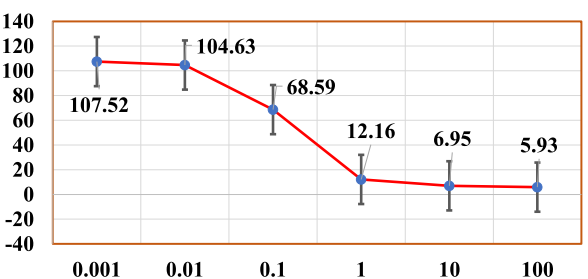

F

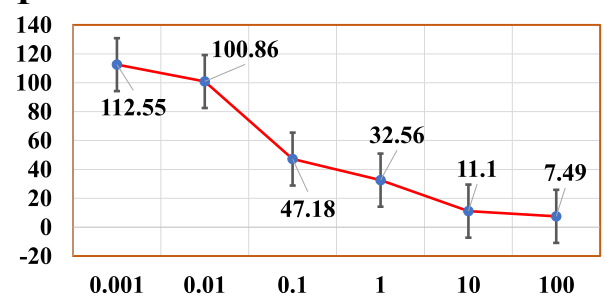

H

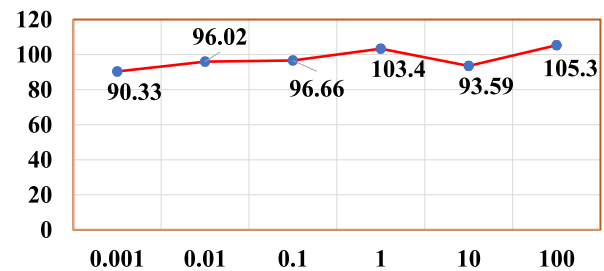

J

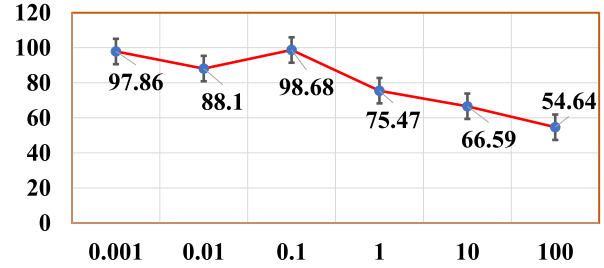

K

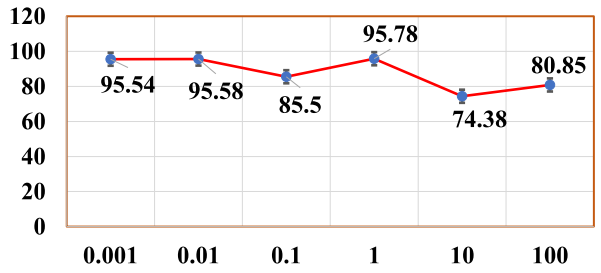

and 8 along bind near to the exit channel below the linker domain in Stage-III, although compound 7 binds to catalytic site in Stage-I. Previous studies also shown that the known compound AZT-TP bind near to the exit channel below the linker domain (Pagadala 2019). Further, the results obtained using regression analysis with $R^{2}=0.73$ shows that binding 
Table. 2 Calculated binding energies $(G$ and $\Delta G)$ and their torsional angles ( $\Phi \mathrm{T}$ and $\Phi \mathrm{P}$ ) for the top seven compounds in the presence of Flu A vRNA that are active against influenza-A virus replication using TCA precipitation assay

\begin{tabular}{llrrrrrr}
\hline Compounds & IUPAC name & $\Phi \mathrm{T}$ & $\Phi \mathrm{P}$ & \multicolumn{1}{l}{$\begin{array}{l}G \\
\mathrm{Kcal} / \mathrm{mol}\end{array}$} & $\begin{array}{l}\Delta G \\
\mathrm{Kcal} / \mathrm{mol}\end{array}$ & $\begin{array}{l}\mathrm{IC}_{25} \\
(\mu \mathrm{M})\end{array}$ & $\begin{array}{l}\mathrm{IC}_{50}(\mu \mathrm{M}) \\
1\end{array}$ \\
\hline & Cyanine 3-Aminoallylcytidine-5'-Triphosphate & -165.2 & 68.3 & -27.2 & 5.2 & 0.03 & 1.69 \\
2 & Cyanine 7-Aminoallyluridine-5'-Triphosphate & 141.9 & -43.9 & -25.6 & 3.6 & 0.88 & 3.58 \\
3 & Cyanine 5-5-Propargylamino-2'-deoxycytidine-5'-Triphosphate & -162.0 & 127.6 & -27.3 & 5.3 & 0.68 & 2.13 \\
4 & Desthiobiotin-6-Aminoallyl-2'-deoxycytidine-5'-Triphosphate & -176.7 & 156.6 & -19.9 & -2.1 & 0.06 & 0.21 \\
5 & N4-Biotin-OBEA-2'-deoxycytidine-5'-Triphosphate & 144.0 & -172.3 & -22.2 & 0.2 & 0.02 & 0.09 \\
6 & P1, P5-Di(adenosine-5') Penta phosphate & -177.1 & -135.2 & -21.5 & -0.5 & 0.03 & 0.09 \\
7 & 5'-Dimethoxytrityl-2'-tosyl-N2-isobutyryl-guanosine & -122.7 & 8.7 & -24 & 2 & 0.25 & 0.68 \\
\hline
\end{tabular}

$\Delta G=G(+$ ve control, $-16 \mathrm{Kcal} / \mathrm{Mol})-G($ drug $)$

Table. 3 Calculated binding energies $(G$ and $\Delta G)$ and their torsional angles ( $\Phi \mathrm{T}$ and $\Phi \mathrm{P})$ for the top seven compounds in the presence of Flu A vRNA with extended primer that are active against influenza-A virus replication using TCA precipitation assay

\begin{tabular}{|c|c|c|c|c|c|c|c|}
\hline Compounds & IUPAC name & $\Phi \mathrm{T}$ & $\Phi P$ & $\begin{array}{l}G \\
\mathrm{Kcal} / \mathrm{mol}\end{array}$ & $\begin{array}{l}\Delta G \\
\mathrm{Kcal} / \mathrm{mol}\end{array}$ & $\begin{array}{l}\mathrm{IC}_{25} \\
(\mu \mathrm{M})\end{array}$ & $\mathrm{IC}_{50}(\mu \mathrm{M})$ \\
\hline 1 & Cyanine 3-Aminoallylcytidine-5'-Triphosphate & -144.2 & 133.8 & -23.4 & 7.6 & 0.03 & 1.69 \\
\hline 2 & Cyanine 7-Aminoallyluridine-5'-Triphosphate & -172.9 & 56.4 & -26.1 & 10.3 & 0.88 & 3.58 \\
\hline 3 & Cyanine 5-5-Propargylamino-2'-deoxycytidine-5'-Triphosphate & -149.9 & 128.7 & -25.6 & 9.8 & 0.68 & 2.13 \\
\hline 4 & Desthiobiotin-6-Aminoallyl-2'-deoxycytidine-5'-Triphosphate & 174.8 & 153.8 & -17.4 & 1.6 & 0.06 & 0.21 \\
\hline 5 & N4-Biotin-OBEA-2'-deoxycytidine-5'-Triphosphate & 131.7 & 174.5 & -21.6 & 5.8 & 0.02 & 0.09 \\
\hline 6 & P1, P5-Di(adenosine-5') Penta phosphate & 152.1 & 60.9 & -26.0 & 10.2 & 0.03 & 0.09 \\
\hline 7 & 5'-Dimethoxytrityl-2'-tosyl-N2-isobutyryl-guanosine & -94.6 & -46.5 & -14.3 & -1.5 & 0.25 & 0.68 \\
\hline
\end{tabular}

$\Delta G=G(+$ ve control, $-15.8 \mathrm{Kcal} / \mathrm{Mol})-G(\mathrm{drug})$

Table. 4 Calculated binding energies $(G$ and $\Delta G$ ) and their torsional angles ( $\Phi \mathrm{T}$ and $\Phi \mathrm{P})$ for the top seven compounds in the presence of Flu B vRNA with extended primer that are active against influenza-A virus replication using TCA precipitation assay

\begin{tabular}{|c|c|c|c|c|c|c|c|}
\hline Compounds & IUPAC name & $\Phi \mathrm{T}$ & $\Phi Р$ & $\begin{array}{l}G \\
\mathrm{Kcal} / \mathrm{mol}\end{array}$ & $\begin{array}{l}\Delta G \\
\mathrm{Kcal} / \mathrm{mol}\end{array}$ & $\begin{array}{l}\mathrm{IC}_{25} \\
(\mu \mathrm{M})\end{array}$ & $\mathrm{IC}_{50}(\mu \mathrm{M})$ \\
\hline 1 & Cyanine 3-Aminoallylcytidine-5'-Triphosphate & 169.2 & 71.5 & -30 & 8 & 0.03 & 1.69 \\
\hline 2 & Cyanine 7-Aminoallyluridine-5'-Triphosphate & 168.6 & 61.6 & -29.5 & 7.5 & 0.88 & 3.58 \\
\hline 3 & Cyanine 5-5-Propargylamino-2'-deoxycytidine-5'-Triphosphate & -159.2 & 98.2 & -27.8 & 5.8 & 0.68 & 2.13 \\
\hline 4 & Desthiobiotin-6-Aminoallyl-2'-deoxycytidine-5'-Triphosphate & 177.5 & 29.8 & -21.0 & -1.0 & 0.06 & 0.21 \\
\hline 5 & N4-Biotin-OBEA-2'-deoxycytidine-5'-Triphosphate & 148.5 & -173.4 & -22.9 & 0.9 & 0.02 & 0.09 \\
\hline 6 & P1, P5-Di(adenosine-5') Penta phosphate & 172.8 & -13.5 & -24.0 & 2 & 0.03 & 0.09 \\
\hline 7 & 5'-Dimethoxytrityl-2'-tosyl-N2-isobutyryl-guanosine & -91.1 & -22.9 & -21.5 & -0.5 & 0.25 & 0.68 \\
\hline
\end{tabular}

$\Delta G=G(+$ ve control, $-13.9 \mathrm{Kcal} / \mathrm{Mol})-G(\mathrm{drug})$

modes of the compounds 1-7 in Stage-I are the most probable conformations that bind to IAV RdRp. Thus, the docked conformations of compounds 1-7 from Stage-I were only discussed further.

Ligand protein interactions show that the compounds 1-3 in Stage-I primarily interact with $\mathrm{PB} 2-\mathrm{N}$, residue nearby motif-II and motif-IV, and the PB1 priming loop that plays an important role in stabilizing the ligand-target interaction. In particular, $\mathrm{Met}^{646}$, $\mathrm{Pro}^{647}$, and $\mathrm{Ala}^{648}$ of the priming loop of PB1 are the key residues stabilizing the compounds 1 and 3 scaffolds within the catalytic site of the IAV RdRp. This binding orientation in turn shields Asp ${ }^{445}$ and $\mathrm{Asp}^{446}$ essential for polymerase activity of influenza virus within the catalytic site (Zamyatkin et al. 2008). These two residues coordinate the metal ions involved in the catalysis. When either of these Asp-Asp residues were 
A

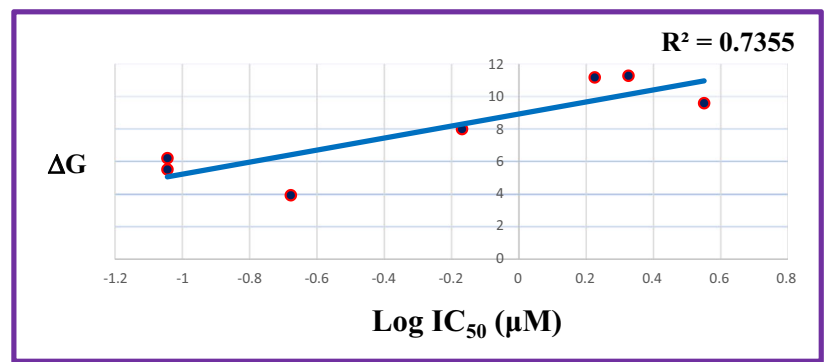

B

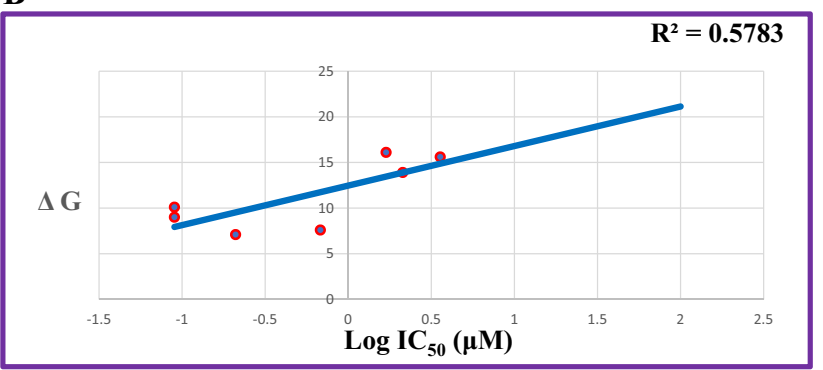

Fig. 6 Regression analysis $\left(R^{2}\right)$ calculated between the $\operatorname{LogIC} \mathrm{C}_{50}$ and $\Delta G$ values predicted in Stage-I (Fig. 6a) and Stage-III (Fig. 6b) for the effective seven compounds that inhibit the IAV RdRp replication in TCA precipitation assay. $\log \mathrm{IC}_{50}$ values are indicated in $X$-axis and their $\Delta G$ values are indicated in $Y$-axis

mutated, Asp ${ }^{445} \mathrm{His}$ and $\mathrm{Asp}^{446} \mathrm{Tyr}$, Asn, or Glu, the catalytic activity of PB1 is completely abolished (Biswas and Nayak 1994). The Asp ${ }^{446}$ may deprotonate the 3 'hydroxy group of the priming NTP by acting as a general base and helps the $\alpha$-phosphate of the NTP to be incorporated into the activator strand. The suitable geometry for the reaction to happen is provided by $\mathrm{His}^{649}$ of the priming loop. Mutational analysis of $\mathrm{His}^{649} \mathrm{~A}$ and $\mathrm{Pro}^{647} \mathrm{~A}$ individually, impair terminal de novo initiation on a vRNA promoter with $\mathrm{P}^{651} \mathrm{~A}$ having the strongest effect (Te Velthuis et al. 2016). To coordinate with the catalytic magnesium ion, the conformational change of $\mathrm{Asp}^{446}$ side chain with $3.5 \AA$ is required to allow the polymerase to initiate polymerization. Apart from these interactions, compounds 1 and 3 have the ability to disturb the structural stability of priming loop by interacting with nearby residues $\mathrm{Pro}^{647}$ and $\mathrm{Ala}^{648}$ of PB1 central domain. However, compound 2 may disturb the structural stability of PB2-N required for subunit binding to PB1-C by interacting with $\mathrm{Asn}^{42}$ and $\mathrm{Ala}^{44}$ of PB2-N. Structural analysis has shown that N-terminus of PB1 established a crucial high-affinity interaction with Cterminal domain of the PA protein (Reuther et al. 2011). Based on the binding modes, it might be speculated that compounds 1-3 may induce some structural changes of motifs-II and IV involved in the formation of catalytic mechanism and putative NTP tunnel formation. Mutational analysis of Lys ${ }^{481}$ of motif-IV to Gln and Arg caused a drastic reduction in catalytic activity of PB1 ranging from 1 to $7 \%$ compared with wild-type which shows the importance of this amino acid in the viral replicase transcriptase activity of PB1 (Biswas and Nayak 1994). The conserved residues of motif D, Lys ${ }^{480}$ and $\mathrm{Lys}^{481}$ involved in NTP binding is further stabilized by contacts with PA helix $\alpha 20$ (656-663) and the PA peptide 671-684 (Pflug et al. 2014). Thus, our compounds 1-3 interferes in stacking interactions between $\mathrm{His}^{649}$, $\mathrm{P}^{651}$ with the incoming NTP by binding to the catalytic site and inhibit the activity of the viral polymerase.

Furthermore, binding mode analysis revealed that compounds 4,5 , and 6 may disturb the structural stability of motif-IV by interacting the nearby residues mainly with $\mathrm{Phe}^{501}$ and $\mathrm{Val}^{502}$ of PB1 central. More likely PB1 Phe $\mathrm{P}^{501}$ plays a main role in IAV RdRp inhibition of these compounds 4, 5, and 6 in tunnel 2 above the PB1 priming loop. However, this residue acts as an electron donor for compound 5 inhibition, while the same residue acts as an electron acceptor for IAV RdRp inhibition by compounds 4 and 6. Plausibly, the side chains of $\mathrm{Arg}^{142}$, $\mathrm{Lys}^{279}$, and Lys $^{670}$ create a favorable environment with a positive electrostatic potential for the binding of these compounds with high potency in TCA precipitation assay. In addition, compounds 4 and 5 might be associated with less conformational changes of PB2 linker domain by tight binding with Lys ${ }^{670}$ of PB2 627 domain. This contact may show the impact on the viral genome transcription and replication by influenza polymerase. These results also reveal that compounds with $2^{\prime}$ deoxycytidine-5'triphosphate and diadenosine Penta phosphate are more potent than other molecules used in the study with 0.09 and $0.21 \mu \mathrm{M}$. This may be due to inhibition of influenza virus RNA polymerase by incorporating in place of CTP and ATP. Previously, McGeoch and Kitron showed that IAV RdRp was stimulated by GpG and GpC (McGeoch and Kitron 1975). Later, plotch and krug had reported that initiation of IAV RdRp cRNA can be stimulated by ApG and GpG dinucleotides which are incorporated at the $5^{\prime}$ end of the cRNA (Plotch and Krug 1977). This enhancement of IAV RdRp by $\mathrm{GpC}$ and $\mathrm{ApG}$ is abolished by compounds 4,5 , and 6 more likely by compounds 5 and 6 than compound 4 in the series. Even though, the binding site of compounds 7 and 8 is the same at the end of the tunnel 2, compound 7 may have a different mechanism of action by donating electrons to $\mathrm{Gly}^{275}$ of linker that leads to the PB2-N2 subdomain and induce conformational changes of PA endonuclease.

By determining the $\mathrm{IC}_{50}$ profiles for each of these seven compounds tested experimentally, it was proved that compounds 1-7 and AZT-TP that was published previously were competitively inhibiting the incorporation of GTP into viral RNA of influenza polymerase (Pagadala 2019). This is in good correlation between our docking studies showing that these compounds bind to catalytic and linker domains 
and prevent the activity of influenza RdRp similar to positive control $2^{\prime}$-deoxy-2'-fluoroguanosine $5^{\prime}$-triphosphate. This also implies that compounds 1-3 may disturb the initiation of replication by inhibiting the incoming NTPs and compounds 4-7 may terminate viral elongation by adding the next correct nucleotide. The results also suggest that the compounds 1-7 after binding to the catalytic site and exit channel inhibit the nucleophilic attack of the $3^{\prime}-\mathrm{OH}$ on the $\alpha$-phosphorous of the incoming NTP. For all the compounds $4,5,6$, and 7 that demonstrated inhibitory activities, the $\mathrm{IC}_{50}$ values were less than onefold better compared with $\mathrm{IC}_{50}$ of $1.66 \mu \mathrm{M}$ of the known compound $2^{\prime}$ deoxy-2'-fluoroguanosine 5'-triphosphate. Even though compounds 8-11 did not inhibit viral replication in TCA precipitation assay, they all bound to the influenza catalytic site. Similar to compounds 1-7, these molecules might not exhibit anti-influenza activity, perhaps due to premature degradation or they may bind away from the catalytic site as explained in Stage-II and III. Structural analysis of these compounds indicates that both indole ring and sulfonyl groups of V-shaped compounds 1-3 might play a major role in binding to the catalytic site of the viral polymerase. Previously, it was also shown that the effect of viral inhibition was high with compound A3 that contains an indole ring when compared with its derivate A3 that lack the indole ring. On the other hand, the high $\mathrm{IC}_{50}$ of linear chain compounds 4 and 5 shows that high flexibility of desthiobiotin and N4-biotin might play an important role in destabilizing PB2 linker of the viral polymerase. In addition, destiobiotin may form destiobiotin-GTP complex through conjugation while N4-biotin may form N4-biotin- $\varepsilon$ Lys $^{670}$ affecting the viral replication in TCA precipitation assay. However, the high $\mathrm{IC}_{50}$ of linear chain compound 6 may be due the presence of di-adenosine that leads to an increase in prolongation of transcription elongation competing with the natural substrates. Considering the binding orientation, it clearly appears that a $\pi$-stacking between $\mathrm{Phe}^{495}$ and $2^{\prime}$-tosyl-N2-isobutyryl-guanosine of compound 7 may play an additional role in the inhibition of viral polymerase activity with an $\mathrm{IC}_{50}$ of $0.68 \mu \mathrm{M}$.

These docking combing through experimental studies clearly indicate that linear chain molecules inhibit polymerase activity with lower $\mathrm{IC}_{50}$ compared with $\mathrm{V}$-shaped and cyclic compounds used in the study. Conceivably, the pharmacophores of these compounds could be useful to screen chemical libraries of nucleoside molecules to identify novel anti-influenza drugs that bind to the catalytic site and still satisfy Lipinski's rule of five. Thus, these findings clearly reveal that these compounds have the strong potential as therapeutic leads for further drug development to treat patients infected with influenza virus.

\section{Conclusion}

In this study, we have successfully combined docking and experimental study to identify eight NTPs that bind to the catalytic site of IAV RdRp from A/PR/8/34/H1N1 strain. Docking studies indicated that linear shape compounds 4,5 , and 6 may induce conformational changes of PB1 motif-IV and PB2 linker domain with an $\mathrm{IC}_{50}$ of 0.21 and $0.09 \mu \mathrm{M}$. Whereas, V-shaped compounds 1, 2, and 3 destabilize the PB1 priming loop involved in initiation of viral replication showing an $\mathrm{IC}_{50}$ of $1.69,3.58$, and $2.13 \mu \mathrm{M}$. However, cyclic compound 8 even though binds nearer to motif-IV of PB1 central domain showed a moderate inhibition with an $\mathrm{IC}_{50}$ of $0.68 \mu \mathrm{M}$. This in turn indicates that the known compound $3^{\prime}$ azido- $3^{\prime}$-deoxy-thymidine- $5^{\prime}$-triphosphate binds to the viral promoter while the positive control $2^{\prime}$ deoxy-2'fluoroguanosine triphosphate binds near to the exit channel inhibiting the transcriptional activity of viral polymerase with the same $\mathrm{IC}_{50}$ of $1.12 \mu \mathrm{M}$ (Pagadala 2019). A good correlation between the $\Delta G$ and $\mathrm{IC}_{50}$ values with an $R^{2}=0.73$ indicate that these molecules bind to the catalytic site inhibiting the incorporation of natural NTPs for replication initiation and elongation of IAV RdRp. The compounds 5 and 6 exhibit significant antiviral effect inhibiting the viral replication with lower $\mathrm{IC}_{50}$ of $0.09 \mu \mathrm{M}$ compared with other compounds used in the study. Thus, this study clearly demonstrates the importance of catalytic site below and above the priming loop for the development of effective anti-influenza drugs. The evaluation of these compounds in further experimental studies will pay the way to develop small molecule therapeutic that can inhibit the IAV viral replication binding to the catalytic site and satisfy Lipinski's rule of five.

\section{Compliance with ethical standards}

Conflict of interest The authors declare that they have no conflict of interest.

Publisher's note Springer Nature remains neutral with regard to jurisdictional claims in published maps and institutional affiliations.

\section{References}

Appleby TC, Perry JK, Murakami E, Barauskas O, Feng J, Cho A, Fox 3rd D, Wetmore DR, McGrath ME, Ray AS, Sofia MJ, Swaminathan S, Edwards TE (2015) Viral replication. Structural basis for RNA replication by the hepatitis $\mathrm{C}$ virus polymerase. Science 347(6223):771-775. https://doi.org/10.1126/science. 1259210

Babar MM, Zaidi NU, Tahir M (2014) Global geno-proteomic analysis reveals cross-continental sequence conservation and druggable sites among influenza virus polymerases. Antivir Res 112:120-131. https://doi.org/10.1016/j.antiviral.2014.10.013 
Biswas SK, Nayak DP (1994) Mutational analysis of the conserved motifs of influenza A virus polymerase basic protein 1. J Virol 68 (3):1819-1826

Butcher SJ, Grimes JM, Makeyev EV, Bamford DH, Stuart DI (2001) A mechanism for initiating RNA-dependent RNA polymerization. Nature 410(6825):235-240. https://doi.org/10.1038/35065653

CDC (2018) Estimated Influenza Illnesses, Medical Visits, Hospitalizations, and Deaths in the United States - 2017-2018 Influenza Season I CDC. https://www.cdc.gov/flu/about/burden/estimates. htm. Accessed 15 Nov 2018

Clark M, Cramer RD, Vanopdenbosch N (1989) Validation of the general-purpose tripos 5.2 force-field. J Comput Chem 10 (8):982-1012. https://doi.org/10.1002/jcc.540100804

Fodor E (2013) The RNA polymerase of influenza a virus: mechanisms of viral transcription and replication. Acta Virol 57 (2):113-122

Furuta Y, Takahashi K, Shiraki K, Sakamoto K, Smee DF, Barnard DL, Gowen BB, Julander JG, Morrey JD (2009) T-705 (favipiravir) and related compounds: Novel broad-spectrum inhibitors of RNA viral infections. Antivir Res 82(3):95-102. https://doi. org/10.1016/j.antiviral.2009.02.198

Ghanem A, Mayer D, Chase G, Tegge W, Frank R, Kochs G, GarciaSastre A, Schwemmle M (2007) Peptide-mediated interference with influenza A virus polymerase. J Virol 81(14):7801-7804. https://doi.org/10.1128/JVI.00724-07

Hengrung N, El Omari K, Serna Martin I, Vreede FT, Cusack S, Rambo RP, Vonrhein C, Bricogne G, Stuart DI, Grimes JM, Fodor E (2015) Crystal structure of the RNA-dependent RNA polymerase from influenza $C$ virus. Nature 527(7576):114-117. https://doi.org/10.1038/nature15525

Ilyushina NA, Hoffmann E, Salomon R, Webster RG, Govorkova EA (2007) Amantadine-oseltamivir combination therapy for H5N1 influenza virus infection in mice. Antivir Ther 12(3):363-370

Influenza (Seasonal) (2018) "Influenza (Seasonal)." https://www.who. $\mathrm{int} /$ news-room/fact-sheets/detail/influenza-(seasonal). Accessed 20 Dec 2018

Kim WY, Young Suh G, Huh JW, Kim SH, Kim MJ, Kim YS, Kim HR, Ryu YJ, Han MS, Ko YG, Chon GR, Lee KH, Choi SH, Hong SB, Korean Society of Critical Care Medicine HNC (2011) Triple-combination antiviral drug for pandemic H1N1 influenza virus infection in critically ill patients on mechanical ventilation. Antimicrob Agents Chemother 55(12):5703-5709. https://doi. org/10.1128/AAC.05529-11

Mackerell Jr. AD, Feig M, Brooks 3rd CL (2004) Extending the treatment of backbone energetics in protein force fields: limitations of gas-phase quantum mechanics in reproducing protein conformational distributions in molecular dynamics simulations. J Comput Chem 25(11):1400-1415. https://doi.org/10.1002/jcc.20065

Martinez E, Marcos MA, Hoyo-Ulloa I, Anton A, Sanchez M, Vilella A, Larrousse M, Perez I, Moreno A, Trilla A, Pumarola T, Gatell JM (2011) Influenza A H1N1 in HIV-infected adults. HIV Med 12 (4):236-245. https://doi.org/10.1111/j.1468-1293.2010.00905.x

McGeoch D, Kitron N (1975) Influenza virion RNA-dependent RNA polymerase: stimulation by guanosine and related compounds. J Virol 15(4):686-695

Metropolis N, Rosenbluth AW, Rosenbluth MN, Teller AH, Teller E (1953) Equation of state calculations by fast computing machines. J Chem Phys 21(6):1087-1092. https://doi.org/10.1063/1.1699114
Pagadala NS (2019) AZT acts as an anti-influenza nucleotide triphosphate targeting the catalytic site of $\mathrm{A} / \mathrm{PR} / 8 / 34 / \mathrm{H} 1 \mathrm{~N} 1 \mathrm{RNA}$ dependent RNA polymerase. J Comput Aided Mol Des. https:// doi.org/10.1007/s10822-019-00189-w

Perez CM, Dominguez MI, Ceballos ME, Moreno C, Labarca JA, Rabagliati R, Vasquez P, Lasso M, Serri M (2010) Pandemic influenza A (H1N1) in HIV-1-infected patients. AIDS 24 (18):2867-2869. https://doi.org/10.1097/QAD.0b013e32833e92d5

Pflug A, Guilligay D, Reich S, Cusack S (2014) Structure of influenza A polymerase bound to the viral RNA promoter. Nature 516 (7531):355-360. https://doi.org/10.1038/nature14008

Plotch SJ, Krug RM (1977) Influenza virion transcriptase: synthesis in vitro of large, polyadenylic acid-containing complementary RNA. J Virol 21(1):24-34

Reich S, Guilligay D, Cusack S (2017) An in vitro fluorescence based study of initiation of RNA synthesis by influenza B polymerase. Nucleic Acids Res 45(6):3353-3368. https://doi.org/10.1093/nar/ gkx043

Reich S, Guilligay D, Pflug A, Malet H, Berger I, Crepin T, Hart D, Lunardi T, Nanao M, Ruigrok RW, Cusack S (2014) Structural insight into cap-snatching and RNA synthesis by influenza polymerase. Nature 516(7531):361-366. https://doi.org/10.1038/ nature 14009

Resa-Infante P, Jorba N, Coloma R, Ortin J (2011) The influenza virus RNA synthesis machine: advances in its structure and function. RNA Biol 8(2):207-215

Reuther P, Manz B, Brunotte L, Schwemmle M, Wunderlich K (2011) Targeting of the influenza A virus polymerase PB1-PB2 interface indicates strain-specific assembly differences. J Virol 85 (24):13298-13309. https://doi.org/10.1128/JVI.00868-11

Seo S, Englund JA, Nguyen JT, Pukrittayakamee S, Lindegardh N, Tarning J, Tambyah PA, Renaud C, Went GT, de Jong MD, Boeckh MJ (2013) Combination therapy with amantadine, oseltamivir and ribavirin for influenza A infection: safety and pharmacokinetics. Antivir Ther 18(3):377-386. https://doi.org/10. 3851/IMP2475

Sherman AC, Mehta A, Dickert NW, Anderson EJ, Rouphael N (2019) The future of flu: a review of the human challenge model and systems biology for advancement of influenza vaccinology. Front Cell Infect Microbiol 9:107. https://doi.org/10.3389/fcimb. 2019.00107

Sidwell RW, Bailey KW, Wong MH, Barnard DL, Smee DF (2005) In vitro and in vivo influenza virus-inhibitory effects of viramidine. Antivir Res 68(1):10-17. https://doi.org/10.1016/j.antiviral.2005. 06.003

Tao Y, Farsetta DL, Nibert ML, Harrison SC (2002) RNA synthesis in a cage-structural studies of reovirus polymerase lambda3. Cell 111(5):733-745

Te Velthuis AJ, Robb NC, Kapanidis AN, Fodor E (2016) The role of the priming loop in influenza A virus RNA synthesis. Nat Microbiol 1(5):16029. https://doi.org/10.1038/nmicrobiol.2016.29

Tisdale M, Ellis M, Klumpp K, Court S, Ford M (1995) Inhibition of influenza virus transcription by 2'-deoxy-2'-fluoroguanosine. Antimicrob Agents Chemother 39(11):2454-2458

Zamyatkin DF, Parra F, Alonso JM, Harki DA, Peterson BR, Grochulski P, Ng KK (2008) Structural insights into mechanisms of catalysis and inhibition in Norwalk virus polymerase. J Biol Chem 283 (12):7705-7712. https://doi.org/10.1074/jbc.M709563200 